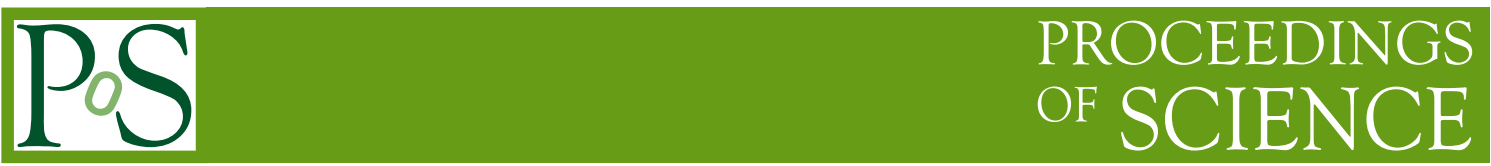

\title{
Nuclear structure and neutrino-nucleus interaction
}

\section{Francisco Krmpotić*}

Instituto de Fisica La Plata, CONICET, Universidad Nacional de La Plata

E-mail: krmpoticafisica.unlp.edu.ar

\section{Nils Paar}

Physics department, Faculty of Science, University of Zagreb, Croatia

E-mail: npaarephy.hr

\section{Arturo R. Samana}

Departamento de Ciências Exactas e Tecnológicas, Universidade Estadual de Santa Cruz, CEP 45662-000 Ilheús, Bahia-BA, Brazil

E-mail: arturo.samana@gmail.com

\section{Carlos A. Bertulani}

Department of Physics, Texas A\&M University Commerce, P.O.3011 Commerce, 75429 TX, USA

E-mail: carlos_bertulani@tamu-commerce.edu

\begin{abstract}
After sketching briefly the formalism for neutrino-nucleus reactions, the following issues are addressed: 1) The consequences of number non-conservation in the Quasiparticle Random Phase Approximation (QRPA) and its inability to reproduce the exclusive weak observables within the triad $\left\{{ }^{12} B,{ }^{12} C,{ }^{12} N\right\}$, 2) The role played by the size of configuration spaces in the Projected QRPA (PQRPA), and the Relativistic QRPA (RQRPA), and 3) The limitations and difficulties of nuclear models in explaining the recently measured quasi-elastic unfolded cross section $\left(v_{\mu},{ }^{12} \mathrm{C}\right)$ at MiniBooNE within the energy range $0.4 \leq E_{v_{\mu}} \leq 1.2 \mathrm{GeV}$.
\end{abstract}

XXXIV edition of the Brazilian Workshop on Nuclear Physics

5-10 June 2011

Foz de Iguaçu, Parana state, Brasil

\footnotetext{
* Speaker.
} 


\section{Introduction}

Recent years have witnessed an intense experimental and theoretical activity oriented towards a better comprehension of neutrino-nucleus interaction. While the main motivation for this task is the demand coming from oscillation experiments in their search for a precise determination of neutrino properties, the relevance of neutrino interaction with matter is more wide-ranging. It is imperative for astrophysics, hadronic and nuclear physics, and physics beyond the standard model. The experimental information on neutrino induced reactions is rapidly growing, and the corresponding theoretical description is a challenging proposition, since the energy scales of interest span a vast region, going from few $\mathrm{MeV}$ for solar neutrinos, to tens of $\mathrm{MeV}$ for the interpretation of experiments with the muon and pion decay at rest and the detection of neutrinos coming from the core collapse of supernova, and to hundreds of $\mathrm{MeV}$ or few $\mathrm{GeV}$ for the detection of atmospheric neutrinos, and for the neutrino oscillation program of the MiniBooNE experiment [1].

The presence of neutrinos, being chargeless particles, can only be inferred by detecting the secondary particles created in colliding and interacting with the matter. Nuclei are often used as neutrino detectors, and in particular ${ }^{12} \mathrm{C}$ which is a component of many scintillator detectors. [2, 3].

Several recent experiments strongly suggest that neutrinos oscillate. This means that a neutrino of a certain flavor (e.g. $v_{\mu}$ ) transforms as it propagates into a neutrino of another flavor (e.g. $\left.v_{e}\right)$ with probability

$$
P_{v_{\mu} \rightarrow v_{e}}=\sin ^{2}(2 \theta) \sin ^{2}\left(1.27 \Delta m^{2} \frac{L_{v}}{E_{v}}\right)
$$

if they are composed of a mixture of mass eigenstates $\left(v_{1}, v_{2}\right)$. Here $\theta$ is the mixing angle between the mass and flavor bases, $\Delta m^{2}=m_{1}^{2}-m_{2}^{2}$ is the $v_{1}$ and $v_{2}$ mass squared differences in $\mathrm{eV}^{2}, L_{v}$ is the baseline, the distance in meters travelled by the neutrino from the source to the detector, and $E_{v}$ is the neutrino energy in $\mathrm{MeV}$.

In the LSND experiment $[2,3]$ the neutrinos $v_{\mu}$ come from the decay of $\pi^{+}$in flight (decay in flight, DIF), whereas the neutrinos $v_{e}$ and the antineutrinos $\bar{v}_{\mu}$ come from the decay of $\mu^{+}$at rest (decay at rest, DAR),

$$
\begin{aligned}
\pi^{+} \rightarrow \mu^{+}+v_{\mu} \quad \pi^{+} \rightarrow & \mu^{+}+v_{\mu} \\
& \downarrow \\
& e^{+}+v_{e}+\bar{v}_{\mu} .
\end{aligned}
$$

DIF DAR

The search for the DAR $\bar{v}_{\mu} \rightarrow \bar{v}_{e}$ oscillations [3] involves the measurement of the reaction $p\left(\bar{v}_{e}, e^{+}\right) n$, which has a large and well known cross section, while the signature for the DIF $v_{\mu} \rightarrow v_{e}$ oscillations [2,3] is marked by the presence in the detector of an isolated high energy electron $\left(60<E_{e}^{\mathrm{DIF}}<200 \mathrm{MeV}\right)$. It is produced by the charge-exchange reaction ${ }^{12} C\left(v_{e}, e^{-}\right){ }^{12} N$, which takes place throughout the tank, the cross section of which $\sigma_{e}$ is established theoretically. The positive LSND results in both channels were interpreted in a two-flavor framework as transitions between the weak eigenstates $v_{\mu}\left(\bar{v}_{\mu}\right)$ and $v_{e}\left(\bar{v}_{e}\right)$ driven by masses and mixing. 
The MiniBooNE experiment was designed to test the LSND signal keeping the same value of $L_{v} / E_{v}$ as LSND:

$$
\begin{array}{clrl}
\text { LSND: } & E_{v} \sim 30 \mathrm{MeV} ; \quad L_{v} \sim 30 \mathrm{~m} ; L_{v} / E_{v} \sim 1, \\
\text { MiniBooNE }: & E_{v} \sim 500 \mathrm{MeV} ; L_{v} \sim 500 \mathrm{~m} ; L_{v} / E_{v} \sim 1 .
\end{array}
$$

The interpretation of neutrino data heavily relies on detailed and quantitative knowledge of the features of the neutrino-nucleus interaction. Detailed comparison between the experimental and theoretical results establish benchmarks needed for verification and/or parameter adjustment of the nuclear models, validity of model approximations, importance of broken symmetries, properness of nuclear forces, and configuration spaces, etc. Having a reliable tool for such calculation is of great importance in a variety of applications, e.g. the neutrino oscillation studies, detection of supernova neutrinos, description of the neutrino transport in supernovae, and the r-process nucleosynthesis. In fact, the uncertainty in the treatment of nuclear effects is now regarded as one of the main sources of systematic uncertainty in neutrino oscillation experiments [4], and quantitative understanding of both exclusive and inclusive weak nuclear response at $0.1 \sim 1.5 \mathrm{GeV}$ is required for data analysis. Moreover, in experimental searches of supernovae neutrino signals is very important the precise knowledge of exclusive cross sections of ${ }^{12} \mathrm{~N}$ and ${ }^{12} \mathrm{~B}$ for establishing the number of detected events during the supernova explosion.

\section{Neutrino (antineutrino)-nucleus cross sections}

We start with the same weak Hamiltonian as the Walecka's group [5], which have developed the most widely used formalism for neutrino-nucleus scattering, i.e.,

$$
H_{W}(\mathbf{r})=\frac{G}{\sqrt{2}} J_{\alpha} l_{\alpha} e^{-i \mathbf{r} \cdot \mathbf{k}},
$$

where $G=(3.04545 \pm 0.00006) \times 10^{-12}$ is the Fermi coupling constant (in natural units), and $k=$ $P_{i}-P_{f} \equiv\left\{\mathbf{k}, i k_{\emptyset}\right\}$ is the momentum transfer. The leptonic current $l_{\alpha} \equiv\left\{\mathbf{l}, i l_{\emptyset}\right\}$ is given by $[6$, Eq. (2.3)], and for the hadronic current operator $J_{\alpha} \equiv\left\{\mathbf{J}, i J_{\emptyset}\right\}$ we use the nonrelativistic form. In spherical coordinates $(m=-1,0,+1)$, and when $\mathbf{k}$ is taken to be along the $z$ axis, it reads $[6,7]$

$$
\begin{aligned}
J_{\emptyset} & =g_{\mathrm{V}}+\left(\bar{g}_{\mathrm{A}}+\bar{g}_{\mathrm{P} 1}\right) \sigma_{0}+i g_{A} \mathrm{M}^{-1} \sigma \cdot \nabla \\
J_{m} & =-g_{\mathrm{A}} \sigma_{m}+m \bar{g}_{\mathrm{W}} \sigma_{m}+\delta_{m 0}\left[-\bar{g}_{\mathrm{V}}+\bar{g}_{\mathrm{P} 2} \sigma_{0}\right]-i g_{V} \mathrm{M}^{-1} \nabla_{m},
\end{aligned}
$$

where the following short notation has been introduced:

$$
\bar{g}_{\mathrm{V}}=g_{\mathrm{V}} \frac{\kappa}{2 \mathrm{M}} ; \bar{g}_{\mathrm{A}}=g_{\mathrm{A}} \frac{\kappa}{2 \mathrm{M}} ; \bar{g}_{\mathrm{W}}=\left(g_{\mathrm{V}}+g_{\mathrm{M}}\right) \frac{\kappa}{2 \mathrm{M}} ; \bar{g}_{\mathrm{P} 1}=g_{\mathrm{P}} \frac{\kappa}{2 \mathrm{M}} \frac{k_{\emptyset}}{\mathrm{m}_{\ell}} ; \bar{g}_{\mathrm{P} 2}=g_{\mathrm{P}} \frac{\kappa}{2 \mathrm{M}} \frac{\kappa}{\mathrm{m}_{\ell}},
$$

with $\mathrm{M}$ and $\mathrm{m}_{\ell}$ being, respectively, the nucleon and charged lepton masses, and $\kappa \equiv|\mathbf{k}|$. The effective vector, axial-vector, weak-magnetism and pseudoscalar dimensionless coupling constants are, respectively

$$
g_{V}=1, g_{A}=1, g_{M}=\kappa_{p}-\kappa_{n}=3.70, g_{P}=g_{A} \frac{2 \mathrm{Mm}_{\ell}}{k^{2}+\mathrm{m}_{\pi}^{2}},
$$


where the estimates for $g_{M}$ and $g_{P}$ come from the conserved vector current (CVC) hypothesis, and from the partially conserved axial vector current (PCAC) hypothesis, respectively. The finite nuclear size (FNS) effect is incorporated via the dipole form factor [8], i.e.,

$$
g \rightarrow g\left[\Lambda^{2} /\left(\Lambda^{2}+k^{2}\right)\right]^{2}, \quad \Lambda=0.85 \mathrm{GeV}
$$

It is convenient define the multipole operators:

$$
\mathrm{O}_{\emptyset \mathrm{J}}=j \mathrm{~J}(\rho) Y_{\mathrm{J} 0}(\hat{\mathbf{r}}) J_{\emptyset} ; \mathrm{O}_{m J}=\sum_{\mathrm{L}} i^{\mathrm{J}-\mathrm{L}} F_{\mathrm{LJ} m} j_{\mathrm{L}}(\rho)\left[Y_{\mathrm{L}}(\hat{\mathbf{r}}) \otimes \mathbf{J}\right]_{J},
$$

where $F_{\mathrm{LJ} m} \equiv(-)^{1+m}(1,-m J m \mid \mathrm{L} 0)$ is a Clebsch-Gordan coefficient. After making use of the CVC the operators $\mathrm{O}_{\alpha\lrcorner} \mathrm{read}[7]$

$$
\begin{aligned}
& \mathrm{O}_{\emptyset \jmath}=g_{V} \mathscr{M}_{\mathrm{J}}^{V}+i g_{A} \mathscr{M}_{J}^{A}+i\left(\bar{g}_{\mathrm{A}}+\bar{g}_{\mathrm{P} 1}\right) \mathscr{M}_{0 \mathrm{~J}}^{A}, \\
& \mathrm{O}_{m\lrcorner}=i\left(\delta_{m 0} \bar{g}_{\mathrm{P} 2}-g_{\mathrm{A}}+m \bar{g}_{\mathrm{W}}\right) \mathscr{M}_{m\lrcorner}^{A}+|m| g_{V} \mathscr{M}_{m J}^{V}+\delta_{m 0} \frac{\tilde{k}_{\emptyset}}{\kappa} g_{\mathrm{V}} \mathscr{M}_{J}^{V},
\end{aligned}
$$

with

$$
\tilde{k}_{\emptyset} \equiv k_{\emptyset}-S\left(\Delta E_{\text {Coul }}-\Delta \mathrm{M}\right) ; \Delta E_{\text {Coul }} \cong \frac{6 e^{2} Z}{5 R} \cong 1.45 Z A^{-1 / 3} \mathrm{MeV}
$$

where the second term in $\tilde{k}_{\emptyset}$ comes from the violation of the CVC by the electromagnetic interaction, $\Delta E_{\mathrm{Coul}}$ is the Coulomb energy difference between the initial and final nuclei, $\Delta \mathrm{M}=$ $\mathrm{M}_{n}-\mathrm{M}_{p}=1.29 \mathrm{MeV}$ is the neutron-proton mass difference, and $S=1(-1)$ for neutrino (antineutrino) scattering. The elementary operators are

$$
\begin{array}{ll}
\mathscr{M}_{\mathrm{J}}^{V}=j_{\mathrm{J}}(\rho) Y_{\mathrm{J}}(\hat{\mathbf{r}}), & \mathscr{M}_{m J}^{A}=\sum_{\mathrm{L} \geq 0} i^{\mathrm{J}-\mathrm{L}-1} F_{\mathrm{LJ} J} j_{\mathrm{L}}(\rho)\left[Y_{\mathrm{L}}(\hat{\mathbf{r}}) \otimes \sigma\right]_{J}, \\
\mathscr{M}_{\mathrm{J}}^{A}=\mathrm{M}^{-1} j_{J}(\rho) Y_{\mathrm{J}}(\hat{\mathbf{r}})(\sigma \cdot \nabla), & \mathscr{M}_{m J}^{V}=\mathrm{M}^{-1} \sum_{\mathrm{L} \geq 0} i^{\mathrm{J}-\mathrm{L}-1} F_{\mathrm{L} J m} j_{\mathrm{L}}(\rho)\left[Y_{\mathrm{L}}(\hat{\mathbf{r}}) \otimes \nabla\right]_{J} .
\end{array}
$$

The transition amplitude $\mathscr{T}_{J_{n}^{\pi}}(\kappa) \equiv \sum_{s_{\ell}, S_{v}}\left|\left\langle\mathrm{~J}_{n}^{\pi}\left|H_{W}(\kappa)\right| 0^{+}\right\rangle\right|^{2}$ for the neutrino-nucleus reaction at a fixed value of $\kappa$, from the ground state $\left|0^{+}\right\rangle$in the $(Z, N)$ target nucleus to the $\mathrm{n}$-th final state $\left|\mathrm{J}_{n}^{\pi}\right\rangle$, with spin $\mathrm{J}$ and parity $\pi$ in the nucleus $(Z \pm 1, N \mp 1)$, reads

$$
\mathscr{T}_{{ }_{n}^{\pi}}(\kappa)=4 \pi G^{2}\left[\sum_{\alpha=\emptyset, 0, \pm 1}\left|\left\langle\mathrm{~J}_{n}^{\pi}|| O_{\alpha \mathrm{J}}(\kappa)|| 0^{+}\right\rangle\right|^{2} \mathscr{L}_{\alpha}-2 \Re\left(\left\langle\mathrm{J}_{n}^{\pi}|| O_{\emptyset \mathrm{J}}(\kappa)|| 0^{+}\right\rangle\left\langle\mathrm{J}_{n}^{\pi}|| O_{0 \mathrm{~J}}(\kappa)|| 0^{+}\right\rangle^{*}\right) \mathscr{L}_{\emptyset 0}\right],
$$

where the momentum transfer is $k=p_{\ell}-q_{v}$, with $p_{\ell} \equiv\left\{\mathbf{p}_{\ell}, i E_{\ell}\right\}, q_{v} \equiv\left\{\mathbf{q}_{v}, i E_{v}\right\}$, and $\ell=e, \mu$. The lepton traces $\mathscr{L}_{\emptyset}, \mathscr{L}_{0}, \mathscr{L}_{ \pm 1}$ and $\mathscr{L}_{\emptyset 0}$ are defined in [6].

The exclusive cross-section (ECS) for the state $\left|\mathrm{J}_{n}^{\pi}\right\rangle$, as a function of the incident neutrino energy $E_{v}$, is

$$
\sigma_{\ell}\left(\mathrm{J}_{n}^{\pi}, E_{V}\right)=\frac{\left|\mathbf{p}_{\ell}\right| E_{\ell}}{2 \pi} F\left(Z+S, E_{\ell}\right) \int_{-1}^{1} d(\cos \theta) \mathscr{T}_{n}^{\pi}(\kappa),
$$


where $E_{\ell}=E_{v}-\omega_{J_{n}^{\pi}}$, and $\left|\mathbf{p}_{\ell}\right|=\sqrt{\left(E_{v}-\omega_{J_{n}}\right)^{2}-m_{\ell}^{2}}$, are the energy and modulus of linear momentum of the lepton $\ell, \omega_{\mathrm{J}_{n}}=-k_{\emptyset}=E_{v}-E_{\ell}$ is the excitation energy of the state $\left|\mathrm{J}_{n}^{\pi}\right\rangle$ relative to the initial state $\left|0^{+}\right\rangle, \kappa=\sqrt{2 E_{v}\left(E_{\ell}-\left|\mathbf{p}_{\ell}\right| \cos \theta\right)-m_{\ell}^{2}+\omega_{J_{n}^{n}}^{2}}$, and $F\left(Z+S, E_{\ell}\right)$ is the Fermi function.

We will also deal with the inclusive cross-sections (ICS),

$$
\sigma_{\ell}\left(E_{v}\right)=\sum_{J_{n}^{\pi}} \sigma_{\ell}\left(\mathrm{J}_{n}^{\pi}, E_{v}\right)
$$

as well as with folded cross-sections, both exclusive, and inclusive

$$
\bar{\sigma}_{\ell}\left(\mathrm{J}_{n}^{\pi}\right)=\int d E_{v} \sigma_{\ell}\left(\mathrm{J}_{n}^{\pi}, E_{v}\right) n_{\ell}\left(E_{v}\right), \bar{\sigma}_{\ell}=\int d E_{v} \sigma_{\ell}\left(E_{v}\right) n_{\ell}\left(E_{v}\right),
$$

where $n_{\ell}\left(E_{v}\right)$ is the neutrino (antineutrino) normalized flux.

In the Extreme Relativistic Limit (ERL), defined by the limit of the lepton velocity $\left|\mathbf{p}_{\ell}\right| / E_{\ell} \rightarrow$ 1, the Eq. (2.12) becomes

$$
\sigma_{\ell}^{E R L}\left(\mathrm{~J}_{n}^{\pi}, E_{v}\right)=\frac{E_{\ell}^{2}}{2 \pi} F\left(Z+S, E_{\ell}\right) \int_{-1}^{1} d(\cos \theta) \mathscr{T}_{J_{n}^{\pi}}^{E R L}(\kappa)
$$

with $\kappa=\sqrt{2 E_{v} E_{\ell}(1-\cos \theta)+\omega_{J_{n}}^{2}}$, and

$$
\begin{aligned}
\mathscr{T}_{\pi}^{E R L}(\kappa) & =4 \pi G^{2}\left[2 \cos ^{2} \frac{\theta}{2}\left|\left\langle\mathrm{~J}_{n}^{\pi}|| \mathrm{O}_{\emptyset J}(\kappa)-\frac{k_{\emptyset}}{\kappa} \mathrm{O}_{0 J}(\kappa) \| 0^{+}\right\rangle\right|^{2}\right. \\
& \left.+\sum_{m= \pm 1}\left|\left\langle\mathrm{~J}_{n}^{\pi}|| \mathrm{O}_{m}(\kappa)|| 0^{+}\right\rangle\right|^{2}\left(\frac{k^{2}}{\kappa^{2}} \cos ^{2} \frac{\theta}{2}+2 \sin ^{2} \frac{\theta}{2}+2 m S \sin \frac{\theta}{2} \sqrt{\frac{k^{2}}{\kappa^{2}} \cos ^{2} \frac{\theta}{2}+\sin ^{2} \frac{\theta}{2}}\right)\right] .
\end{aligned}
$$

\section{Nuclear Structure}

The nuclear Hamiltonian is of the form $H=H_{m f}+V_{\text {res }}$, where $H_{m f}$ is the mean field Hamiltonian, i.e., one-body central field, and $V_{\text {res }}$ is the residual interaction, which is assumed to be small. The later causes long-range particle-hole (ph), and short-range particle-particle (pp) correlations.

The nuclear models that are used for describing the neutrino-nucleus cross reactions can be grouped into:

a) Shell Model (SM), which mostly accounts for the pp correlations,

b) Tamm-Dancoff Approximation (TDA), which considers only the forward-going ph correlations,

c) Random Phase Approximation (RPA), and its continuum version CRPA, engender both forwardand backward-going ph correlations,

d) Quasiparticle RPA (QRPA), which deals with ph correlations, and partially also with pp correlations, but does not conserve the number of particles,

e ) Relativistic QRPA (RQRPA), which is modern version of the QRPA,

f) Projected QRPA (PQRPA), which includes the same correlations as the QRPA, but conserves the number of particles, and

g ) Fermi Gas Model (FGM), which is the simplest mean field of nuclear physics. One treats nucleons as non-interacting nuclear matter in the limit that the radius of the nucleus becomes infinite, and the Pauli exclusion principle is obeyed. In the simplest version no residual interaction is considered. 
In all finite nuclear structure calculations, not only the nuclear Hamiltonian but also the size of the configuration space plays an important role. Henceforth a single-particle (s.p.) space that includes all orbitals within $N$ harmonic oscillator (HO) shells will be labeled as space $S_{N}$.

The main issues addressed here are: 1) The consequences of number non-conservation in QRPA, 2) The dependence of the $v-{ }^{12} \mathrm{C}$ cross-section on the size of configuration spaces in PQRPA and RQRP, and 3) The ability of nuclear models to explain the the MiniBooNE experiment [1]. The nuclear model that will be used are:

- PQRPA, where the mean field is described by the HO with $N=2,3,4,6$, and the residual interaction is a simple $\delta$-force $V=-4 \pi\left(v_{s} P_{s}+v_{t} P_{t}\right) \delta(r)$, where $t=v_{t}^{p p} / v_{s}^{\text {pair }}\left(v_{s}^{\text {pair }}=\right.$ $\left.\left(v_{s}^{\text {pair }}(p)+v_{s}^{\text {pair }}(n)\right) / 2\right)$ is taken to be a free parameter [7]. For the spaces $S_{2}, S_{3}$, and $S_{4}$ the s.p. energies and pairing strengths $v_{s}^{\text {pair }}(p)$, and $v_{s}^{\text {pair }}(n)$ were varied in a $\chi^{2}$ search to account for the experimental spectra of odd-mass nuclei ${ }^{11} \mathrm{C},{ }^{11} \mathrm{~B},{ }^{13} \mathrm{C}$, and ${ }^{13} \mathrm{~N}$, as explained in Ref. [6]. This method, however, is not applicable for the space $S_{6}$ which comprises 21 s.p. levels. Therefore in this case the energies were derived in the way done in Ref. [9], while the pairing strengths were adjusted to reproduce the experimental gaps in ${ }^{12} \mathrm{C}$, considering all the quasiparticle energies up to $100 \mathrm{MeV}$.

- RQRPA where both the mean field and the residual interaction are derived from the same effective Lagrangian density [9]. The ground state is calculated in the Relativistic HartreeBogoliubov (RHB) model using effective Lagrangians with density dependent meson-nucleon couplings and DD-ME2 parameterization, and pairing correlations are described by the finite range Gogny force. The HO basis with $N=20$ or $N=30$ is used only in the RHB calculation in order to determine the ground state and the single-particle spectra. The wave functions employed in RPA equations are obtained by converting the original basis to the coordinate representation, and the size of the RQRPA configuration space is limited by $2 q p$ energy cut-offs $E_{2 q p}$.

In the evaluation of both neutrino, and antineutrino ICS the summation in (2.13) goes over all $n$ states with spin and parity $\mathrm{J}^{\pi} \leq 7^{ \pm}$in the PQRPA, and over all $\mathrm{J}^{\pi} \leq 14^{ \pm}$in the RQRPA.

\subsection{QRPA versus PQRPA}

The experimental data for the exclusive and inclusive cross sections, given in Table 1, show that the DAR and DIF processes are of quite different nature: while the first one is dominated in proportion of $2 / 3$ by the Gamow-Teller (GT) transition to the ground state $1_{1}^{+}$in ${ }^{12} N$, the second one populates almost entirely the excited states through the forbidden transitions. It is quite a difficult task for the nuclear structure models to describe both cross sections simultaneously. The SM treats correctly the Pauli Principle within the $p$-shell, which is crucial for the correct distribution of the GT strength, whereas the predictions for high-lying states are less certain because of the truncation of the model space. In fact, the SM calculation performed by Hayes and Towner [11] reproduces fairly well several data. But, in a later SM study, Volpe et al. [12] noted that this concordance could be an artifact because the employed model space was not large enough to exhaust the charge-exchange sum rules. More, the same authors have shown that when a more extended space is employed the SM cross sections are increased exceeding the experimental LSND result. The 
Table 1: Calculated and experimental flux-averaged exclusive $\bar{\sigma}_{\ell}\left(1_{1}^{+}\right)$, and inclusive $\bar{\sigma}_{\ell}$ cross section for the ${ }^{12} C\left(v_{e}, e^{-}\right)^{12} N$ DAR reaction (in units of $\left.10^{-42} \mathrm{~cm}^{2}\right)$ and for the ${ }^{12} C\left(v_{\mu}, \mu^{-}\right){ }^{12} N$ DIF reaction (in units of $\left.10^{-40} \mathrm{~cm}^{2}\right)$.

\begin{tabular}{l|cccc}
\hline & $\bar{\sigma}_{e}\left(1_{1}^{+}\right)$ & $\bar{\sigma}_{e}$ & $\bar{\sigma}_{\mu}\left(1_{1}^{+}\right)$ & $\bar{\sigma}_{\mu}$ \\
\hline Exp. [10] & $8.9 \pm 0.3 \pm 0.9$ & $13.2 \pm 0.4 \pm 0.6$ & $0.56 \pm 0.08 \pm 0.10$ & $10.6 \pm 0.3 \pm 1.8$ \\
\hline SM [11] & 7.9 & 12.0 & 0.56 & 13.8 \\
SM [12] & 8.4 & 16.4 & 0.70 & 21.1 \\
PQRPA [6] & 8.1 & 18.6 & 0.59 & 13.0 \\
RPA [12] & 49.5 & 55.1 & 2.09 & 19.2 \\
CRPA [13] & 38.4 & 44.3 & 3.11 & 22.8 \\
QRPA [12] & 42.9 & 52.0 & 1.97 & 20.3 \\
\hline
\end{tabular}

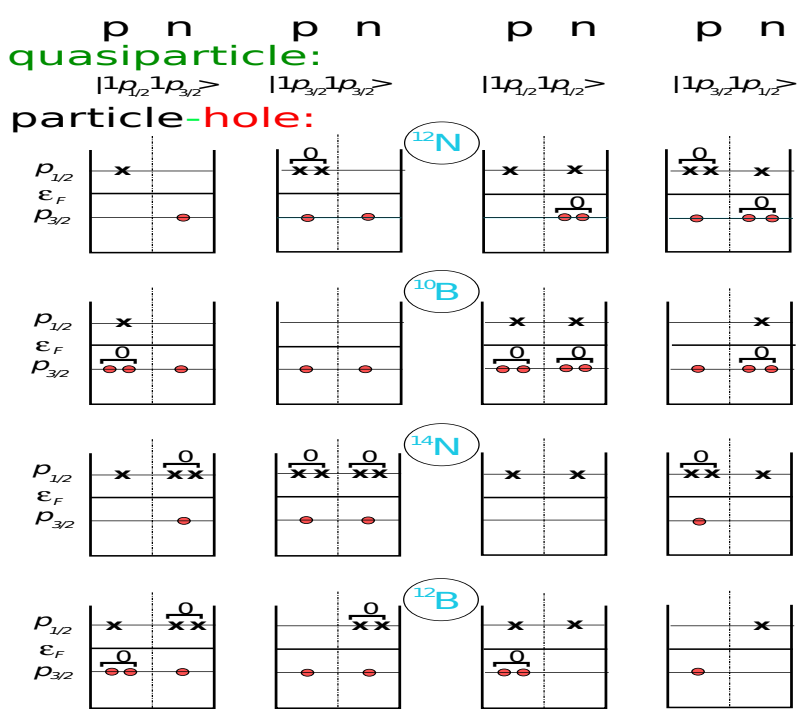

Figure 1: Schematic representation of the particle-hole compositions for proton-neutron quasiparticle states $\left|p_{1 / 2}^{\pi} p_{3 / 2}^{v}\right\rangle$, $\left|p_{3 / 2}^{\pi} p_{3 / 2}^{v}\right\rangle,\left|p_{1 / 2}^{\pi} p_{1 / 2}^{v}\right\rangle$, and $\left|p_{3 / 2}^{\pi} p_{1 / 2}^{v}\right\rangle$. The zero-angular-momentum couplings of $2 \mathrm{p}$ or $2 \mathrm{~h}$ are indicated by a horizontal bracket. $\varepsilon_{F}$ represents the Fermi energy.

RPA like models include high-lying ph excitations, but very frequently completely fail to account for the amount and distribution of the GT strength as can be seen from Table 1. This is the reason why the CRPA is unable to explain the weak processes ( $\beta$-decays, $\mu$-capture, and neutrino induced reactions) among the ground states of the triad $\left\{{ }^{12} B,{ }^{12} C,{ }^{12} N\right\}$ : a rescaling factor of the order of 4 is needed to bring the calculations and the data to agree [13]. On the other hand, the inability of the QRPA to reproduce the exclusive observables is caused by the violation of the number of particles in the BCS approximation, which is not important in the solid state physics where one deals with $\sim 10^{23}$ particles, but is quite relevant in nuclear physics. This symmetry is restored by the number projection procedure that is done within the PQRPA, and this is the reason for the huge differences between the QRPA and PQRPA values for the ECS listed in Table 1. What makes the projection is illustrated in Fig. 1, where the particle-hole composition of proton-neutron quasiparticle states 
$\left|p_{1 / 2}^{\pi} p_{3 / 2}^{v}\right\rangle,\left|p_{3 / 2}^{\pi} p_{3 / 2}^{v}\right\rangle,\left|p_{1 / 2}^{\pi} p_{1 / 2}^{v}\right\rangle$, and $\left|p_{3 / 2}^{\pi} p_{1 / 2}^{v}\right\rangle$ is shown. While the QRPA does not differentiate between the 4 nuclei $\left({ }^{12} \mathrm{~N},{ }^{10} \mathrm{~B},{ }^{14} \mathrm{~N}\right.$, and $\left.{ }^{12} \mathrm{~B}\right)$, embracing all np-nh configurations shown in the 4 rows of the figure, the PQRPA includes only the first (last) row in the case of ${ }^{12} \mathrm{~N}\left({ }^{12} \mathrm{~B}\right)$, being the key components in their wave functions those corresponding to $1 \mathrm{p}-1 \mathrm{~h}$ configurations, i.e., the first for ${ }^{12} \mathrm{~N}$, and the last for ${ }^{12} \mathrm{~B}$; see Eqs. (3.1), (3.2) and (3.3) in Ref. [7]. From now on only the PQRPA should be used for the ECSs.

\subsection{Size of the configuration space}
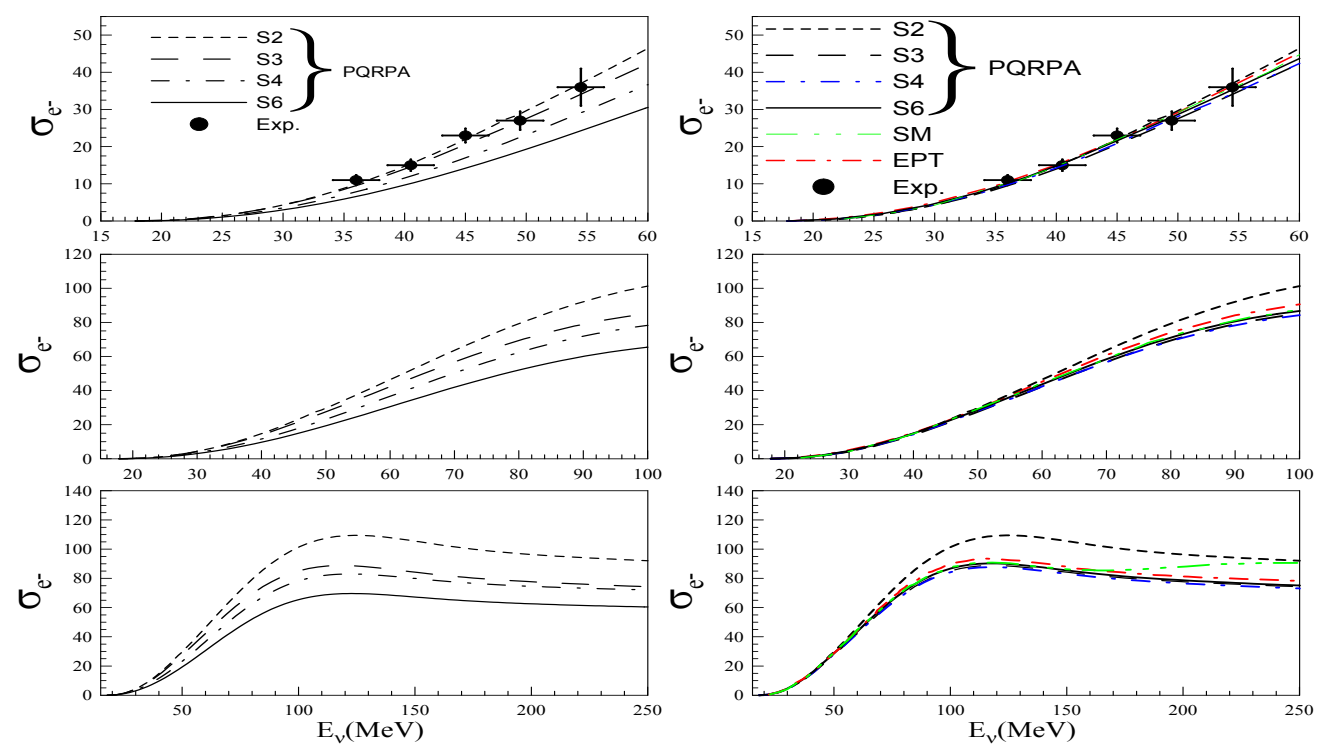

Figure 2: (Color online) Exclusive cross section $\sigma_{e^{-}}\left(1_{1}^{+}, E_{V}\right)$ for the reaction ${ }^{12} \mathrm{C}\left(v_{e}, e^{-}\right){ }^{12} \mathrm{~N}$ (in units of $10^{-42} \mathrm{~cm}^{2}$ ), as a function of the incident neutrino energy $E_{v}$, when evaluated with the PQRPA. On the left side $t=0$ for all $S_{N}$, whereas on the right side $t=0$ for $S_{2}$, and $S_{3}, t=0.2$ for $S_{4}$, and $t=0.3$ for $S_{6}$. The SM, and EPT calculations are, respectively, from Refs. [14], and [15]. The experimental data in the DAR region are from Ref. [16].

The ${ }^{12} \mathrm{C}\left(v_{e}, e^{-}\right){ }^{12} \mathrm{~N}\left(1_{1}^{+}\right)$ECS evaluated with the PQRPA is plotted as a function of the incident neutrino energy $E_{v}$ in Fig. 2 for: i) $\pi^{+}$-decay at rest (DAR) region $\left(E_{v} \leq 60 \mathrm{MeV}\right)$, where the ECS was measured [16], ii) region where the supernovae neutrino signal is expected $\left(E_{v} \leq 100 \mathrm{MeV}\right)$, and iii) $\pi^{+}$(DIF)-decay at flight region, where the neutrinos oscillation search was done in LSND $\left(E_{v} \leq 250 \mathrm{MeV}\right)$. On the left side is $t=0$ for all spaces $S_{N}$, whereas on the right side $t$ is gauged to reproduce the ground state energy of ${ }^{12} \mathrm{~N}$ and the $B(G T)$-values of ${ }^{12} \mathrm{~N}$ and ${ }^{12} \mathrm{~B}$, getting $t=0$ for $S_{2}$ and $S_{3}, t=0.2$ for $S_{4}$, and $t=0.3$ for $S_{6}$. Thus, to obtain the agreement with the experimental data it is necessary to increase $t$ when the size of the space is increased. This change of parametrization hint at the self-consistency of the PQRPA, and comes from the fact that in this model: i) the GT strength allocated in the ground state is moved to another $1^{+}$states when the size of the space is increased, and ii) the effect of the $p p$ residual interaction goes in the opposite direction, returning the GT strength to the $1_{1}^{+}$state. In the same figure are exhibited as well the results for the ECSs evaluated within the SM [14], and the Elementary Particle Treatment (EPT) [15]. Both of them agree well with the data and with our calculation. 

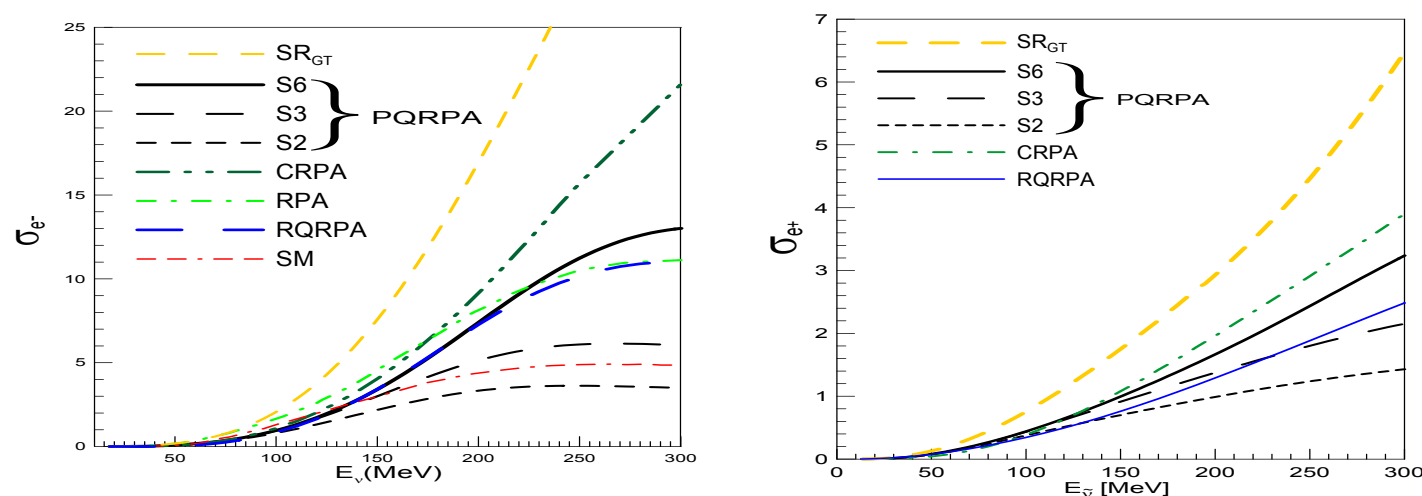

Figure 3: (Color online) Inclusive cross sections $\sigma_{e^{-}}\left(E_{V}\right)$, and $\sigma_{e^{+}}\left(E_{\tilde{V}}\right)$ (in units of $10^{-39} \mathrm{~cm}^{2}$ ) for the reactions ${ }^{12} \mathrm{C}\left(v_{e}, e^{-}\right){ }^{12} \mathrm{~N}$, and ${ }^{12} \mathrm{C}\left(\tilde{v}_{e}, e^{+}\right){ }^{12} \mathrm{~B}$, respectively, plotted as a function of incident neutrino and antineutrino energies. The GT sum rule limit $S R_{G T}$ [7] and several previous RPA-like calculations, namely: RPA [12], CRPA [17], and RQRPA within $S_{20}$ for two-quasiparticle cutoff $E_{2 q p}=100 \mathrm{MeV}$ [9], PQRPA within $S_{2}, S_{3}$, and $S_{6}$ [7], as well as the shell model result [12], are also exhibited.

Many SM and RPA-like calculations of the ICS $\sigma_{e^{-}}\left(E_{V}\right)$, employing different effective axialvector coupling constants, and different s.p. spaces, have been done so far. A few of them are exhibited in the left panel of Fig. 3, namely: a) SM and RPA [12], with $g_{A}=0.88$, and $S_{3}$, b) CRPA [17], with $g_{A}=1.26$, and $S_{4}$, c) RQRPA [9], with $g_{A}=1.23, S_{20}$, and $E_{2 q p}=100 \mathrm{MeV}$, and d) PQRPA [7], with $g_{A}=1.0, S_{2}, S_{3}$, and $S_{6}$. In spite of very significant differences in $g_{A}$, and the s.p. spaces, the different calculations of $\sigma_{e^{-}}\left(E_{v}\right)$ yield quite similar results for energies $E_{v} \lesssim 130 \mathrm{MeV}$. But for higher energies they could become quite different, and are clearly separated in two groups at $E_{v}=300 \mathrm{MeV}$. In the first group with $\sigma_{e^{-}}\left(E_{v}\right) \lesssim 5 \times 10^{-39} \mathrm{~cm}^{2}$ are: the SM, and PQRPA within spaces $S_{2}, S_{3}$, while in the second one with $\sigma_{e^{-}}\left(E_{V}\right) \gtrsim 10 \times 10^{-39} \mathrm{~cm}^{2}$ are: the RPA, RQRPA, CRPA and PQRPA within space $S_{6}$. Volpe et al. [12] have found that the difference between their SM and RPA calculations is due to differences in the correlations taken into account, and to a too small SM space. Similar results for the inclusive ${ }^{12} \mathrm{C}\left(\tilde{v}, e^{+}\right){ }^{12} \mathrm{~B}$ cross-section $\sigma_{e^{+}}\left(E_{\tilde{v}}\right)$ are displayed in the right panels of Fig. 3, and analogous comments can be done here. For the comparison, we show in the figure the antineutrino- ${ }^{12} \mathrm{C}$ cross-sections evaluated with the CRPA [17]. As there are no experimental data on flux unfolded ICSs for $E_{v} \leq 400 \mathrm{MeV}$ we cannot conclude which of the results displayed in Fig. 3 are good and which are not. We can only infer that the ICSs strongly depend on the size of the s.p. space. In the PQRPA calculations we were not able to use spaces lager than $S_{6}$ because of numerical difficulties. Thus all results that follow were done with the RQRPA where such calculations are feasible [7].

The effect of the cut-off energy within the $S_{20}$, and $S_{30}$ spaces on $\sigma_{e^{-}}\left(E_{V}\right)$ for $E_{V}$ up to 600 $\mathrm{MeV}$ is shown in the Figure 4. By comparing the two panels it is easy to figure out that up to $E_{2 q p}=300 \mathrm{MeV}$ the cross sections obtained with the two spaces are basically the same. Small differences between in the cross sections obtained using the $S_{20}$ versus the $S_{30}$ spaces for $E_{2 q p}$ up to $300 \mathrm{MeV}$ are caused by modifications of positive-energy single-particle states contributing to the RQRPA configuration space within the restricted $2 q p$ energy window. But, for $E_{V} \gtrsim 400$ $\mathrm{MeV}$ additional transition strength appears in the $S_{30}$ space when $E_{2 q p}$ is moved up to $400 \mathrm{MeV}$, after which further increase in $E_{2 q p}$ has a very small effect. It was concluded therefore [7] that 


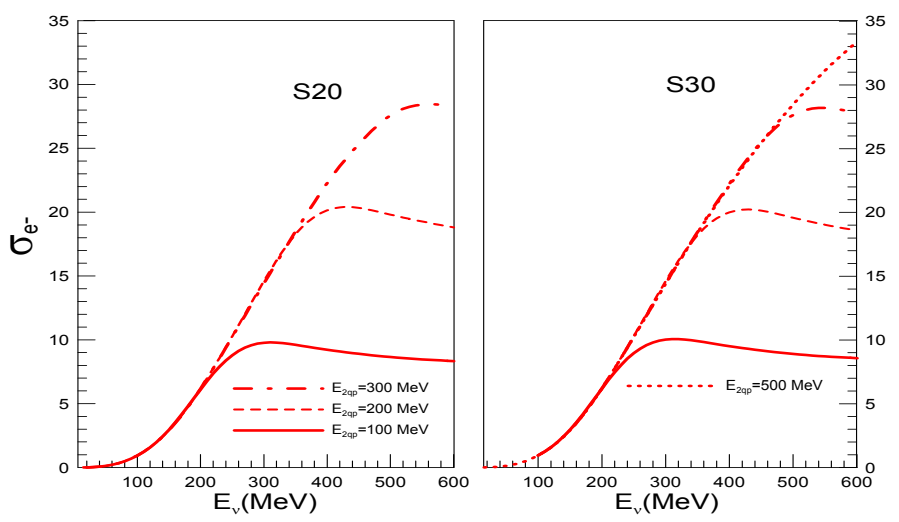

Figure 4: (Color online) Inclusive ${ }^{12} \mathrm{C}\left(v, e^{-}\right){ }^{12} \mathrm{~N}$ cross-section $\sigma_{e^{-}}\left(E_{v}\right)$ (in units of $\left.10^{-39} \mathrm{~cm}^{2}\right)$ plotted as a function of neutrino energy $E_{v}$, evaluated in RQRPA with different configuration spaces and different $E_{2 q p}$ quasiparticle cut-off energies within $S_{20}$, and $S_{30}$ s.p. spaces.

the configuration space engendered by $N=20 \mathrm{HO}$ shells with $E_{2 q p}=300 \mathrm{MeV}$, is large enough to describe $\sigma_{e^{-}}\left(E_{v}\right)$ with $E_{v}$ up to $400 \mathrm{MeV}$. Similarly, the space brought about by $N=30 \mathrm{HO}$ shells with $E_{2 q p}=400 \mathrm{MeV}$ is appropriate to account for $\sigma_{e^{-}}\left(E_{V}\right)$ up to $E_{v}=600 \mathrm{MeV}$. For larger neutrino energies very likely we would have to continue increasing the number of shells.

\subsection{MiniBooNE experiment}

The recently measured quasi-elastic $(\mathrm{QE})$ unfolded cross section $\left(v_{\mu},{ }^{12} \mathrm{C}\right)$ at MiniBooNE [1] within the energy range $0.4 \leq E_{v_{\mu}} \leq 1.2 \mathrm{GeV}$ turned out to be much higher than the FGM calculation with the axial vector constant $g_{A}=1.26$, and cutoff $\Lambda_{A}=1.03$. (frequently called axial mass $M_{A}$ [19]), as shown in Fig. 5. This has caused great concern and originated an extensive debate, which is still going on. There are basically two proposals to brings the FGM into agreement with the data: 1) an anomalously large $\Lambda_{A}=1.35$ [1], and 2) the addition of the np-nh excitations, coming from the ground state correlations (GSC), to the 1p-1h FGM [18]. Some caution should be expressed, however, before drawing definitive conclusions from the agreements with data. The first proposal is difficult to understand theoretically and it is $\sim 30 \%$ larger than NOMAD data at $5-10 \mathrm{GeV}$ [19]. Regarding the second one it should be kept in mind that the multi ph excitations are always taken into account in the QRPA calculations. (Some of them are illustrated in Fig. 1). Here, however, they don't give rise to any additional transition strength, but they only redistribute it. The same happens in the SM, where the np-nh excitations correspond to the "configuration admixtures". Thus, as pointed out in Ref. [7], the increase of the transition strength by the np-nh excitations could be just an artifact of the FGM, induced by the lack of an appropriate normalization of the ground state wave function; see also Refs. [20,21]. Strictly speaking Martini et al. [18] also include in their calculations the exchange currents that are genuine two-body operators and are not considered in the RQRPA calculations. Yet, Alberico et al. [22] have shown that their effect is relatively small in comparison with that induced by the GSC.

In view of above scenario we wanted to see what the RQRPA could tell us regarding the QE $\left(v_{e},{ }^{12} \mathrm{C}\right.$ ) cross section when evaluated within $S_{30}$ (with $E_{2 q p}=500 \mathrm{MeV}$ ), $g_{A}=1.26$, and cutoff $\Lambda_{A}=1.03$. The corresponding results are displayed in Fig. 5, where the agreement between the 


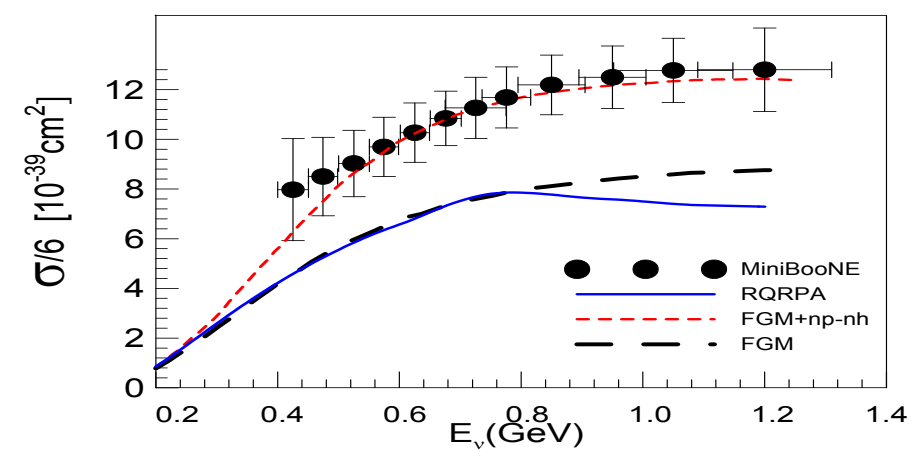

Figure 5: (Color online) Quasi-elastic $\left(v_{\mu},{ }^{12} \mathrm{C}\right)$ MiniBooNE cross section [1], compared with the FGM results with and without np-nh excitations [18], and the RQRPA calculation within $S_{30}$ space, and $E_{2 q p}=500$ MeV. In all cases is $g_{A}=1.26$, and $\Lambda_{A}=1.03$.

PQRPA and FGM cross sections for $E_{v_{\mu}}$ up to $\sim 0.8 \mathrm{GeV}$ deserves to be mentioned, as well as that for higher neutrino energies the PRRPA cross section is smaller. This could indicate that: a) the kinematics play the dominant role for the ICS, while the dynamics is only relevant for the ECS, and b) by increasing the number of HO shells the PQRPA result would probably reach the FGM one also for $E_{v_{\mu}}>0.8 \mathrm{GeV}$, but it would never account for the experimental data.

\section{Final Remarks}

For $E_{v_{e}} \lesssim 50 \mathrm{MeV}$, the exclusive cross section dominates over the inclusive one, and decreases with the size of the configuration space; appropriate models are the SM and the PQRPA. Above $E_{V_{e}} \gtrsim 50 \mathrm{MeV}$, dominates the inclusive cross section, which strongly increases with the size of the configuration space; we cannot say which nuclear model is the most suitable. The number of harmonic oscillator shells should be increased above $N=30$ for $E_{v}>600 \mathrm{MeV}$. None of the nuclear models (SM, RPA, QRPA,PQRPA, RQRPA) is able to account for the MiniBooNE measurement, which can only be described by the FGM, either by introducing an anomalously large axial mass $\left(\Lambda_{A}=1.35\right)$, or by including the np-nh excitations. Yet, none of these two ways out of the puzzle is fully satisfactory, and we are likely to agree with Benhar [23] in the sense that a new paradigm is needed for modeling the neutrino-nucleus interaction.

\section{References}

[1] A. A. Aguilar-Arevalo, et al. (MiniBooNE Collaboration), Phys. Rev. D 81, 0292005 (2010).

[2] C. Athanassopoulus, et al. [LSND Collaboration], Phys. Rev. Lett. 81, 1774 (1998).

[3] A. Aguilar et al. [LSND collaboration], Phys. Rev. D 64, 112007 (2001).

[4] A. Samana, F. Krmpotić, A. Mariano and R. Zukanovich Funchal, Phys. Lett. B642, 100 (2006).

[5] J.D. Walecka, Theoretical Nuclear and Subnuclear Physics, Oxford University Press, NY, (1995). 
[6] F. Krmpotić, A. Samana, and A. Mariano, Phys. Rev. C 71, 044319 (2005).

[7] A.R. Samana, F. Krmpotić, N. Paar, and C.A. Bertulani, Phys. Rev. C 83, 024303 (2011).

[8] T. Kuramoto, M.Fukugita, Y. Kohyama, and K. Kubodera, Nucl. Phys. A512, 711 (1990).

[9] N. Paar, D. Vretenar, T. Marketin, and P. Ring, Phys. Rev. C 77024608 (2008).

[10] L. B. Auerbach et al. , [LSND Collaboration], Phys. Rev. C 66, 015501 (2002).

[11] A.C. Hayes and I.S. Towner, Phys. Rev. C 61, 044603 (2000).

[12] C. Volpe, N. Auerbach, G. Colò, T. Suzuki, N. Van Giai, Phys. Rev. C 62, 015501 (2000).

[13] E. Kolbe, K. Langanke and S. Krewald, Phys. Rev. C 49, 1122 (1994).

[14] J. Engel, E. Kolbe, K. Langanke,and P. Vogel, Phys. Rev. C 54, 2740 (1996).

[15] M. Fukugita, Y. Kohyama and K. Kubodera, Phys. Lett. B212, 139 (1988).

[16] Athanassopoulus C et al. [LSND Collaboration] 1997 Phys. Rev. C 552078

[17] E. Kolbe, K. Langanke and P. Vogel Nucl. Phys. A652, 91 (1999).

[18] M. Martini, M. Ericson, G. Chanfray and J. Marteau, Phys. Rev. C 80, 065501 (2009), and Phys. Rev. C 81, 045502 (2010).

[19] V. Lyubushkin et al. (NOMAD Collaboration), Eur. Phys. J. C 63 (2009) 355.

[20] D. Van Neck, M. Waroquier, V. Van der Sluys, and J. Ryckebusch, Phys. Lett. B274, 142(1992).

[21] A. Mariano, F. Krmpotić, and A.F.R. de Toledo Piza, Phys. Rev. C 53, 1664 (1996).

[22] W. M. Alberico, M. Ericson, and A. Molinari, Ann. Phys. 154, 356 (1984).

[23] O. Benhar, arXiv:1012.2032, Nucl. Phys B Proc. Suppl. 00 (2010) 1. 